\title{
Resistencias de $M$. tuberculosis complex en Navarra: diferencias entre población autóctona e inmigrante (2000-2007)
}

\author{
M. tuberculosis complex drug resistance in Navarre: differences \\ between the local and immigrant populations
}

\author{
A. Navascués ${ }^{1}$, I. Otamendi ${ }^{1}$, L. Torroba ${ }^{2}$, A. I. Álvaro ${ }^{1}$, M. Ojer ${ }^{1}$, A. Ruz ${ }^{1}$, J. Castilla ${ }^{3}$
}

\section{RESUMEN}

Fundamento. La tuberculosis es un importante problema de salud pública, cuya epidemiología ha cambiado en los últimos años en nuestro país debido al aumento de población inmigrante. El objetivo de este trabajo ha sido valorar la frecuencia de resistencias a los cuatro antituberculosos principales, tanto en población autóctona como inmigrante.

Material y métodos. Se han estudiado los antibiogramas de 457 aislamientos de Mycobacterium tuberculosis realizados en el Hospital de Navarra en el periodo 20002007. Los antibiogramas fueron procesados mediante el sistema BACTEC460TB.

Resultados. El 26,3\% de las cepas correspondieron a inmigrantes, produciéndose a lo largo del periodo un incremento significativo. Las frecuencias de resistencias a los distintos fármacos antituberculosos en población autóctona e inmigrante, respectivamente fueron: a al menos uno $5,6 \%$ vs $20,8 \%$ (p $<0,001$ ), a isoniacida $4,5 \%$ vs $14,2 \%$ ( $\mathrm{p}<0,001$ ), a estreptomicina $2,4 \%$ vs $12,5 \%$ (p $<0,001)$, a rifampicina $0,9 \%$ vs $5 \%(\mathrm{p}<0,05)$, a etambutol $0,3 \%$ vs $2,5 \%(\mathrm{p}<0,05)$ y multirresistencia $0,3 \%$ vs $2,5 \%$ $(\mathrm{p}<0,05)$.

Conclusión. Existen diferencias significativas entre la población autóctona e inmigrante en el patrón de resistencias de las cepas aisladas. Esta última, debido a la alta frecuencia con que presenta resistencia a la isoniacida, debe tratarse inicialmente con una pauta de cuatro fármacos hasta disponer del resultado del antibiograma.

Palabras clave. M. tuberculosis. Resistencia. Inmigración.

\begin{abstract}
Background. Tuberculosis is an important public health problem, whose epidemiology in our country has changed in recent years due to the increase in the immigrant population. The aim of this article is to evaluate the frequency of resistance to the four principal antitubercular drugs in both the local and immigrant populations.
\end{abstract}

Methods. A study was made of the antibiograms of 457 isolations of Mycobacterium tuberculosis carried out in the Hospital of Navarre in 2000-2007. The antibiograms were processed using the BACTEC460TB system.

Results. Twenty six point three percent of the strains corresponded to immigrants, with a significant increase occurring over the period. The frequencies of resistances to the different antitubercular drugs in the local and immigrant populations respectively were: to at least one $5.6 \%$ vs $20.8 \%$ ( $<<0.001$ ); to isoniazid $4.5 \%$ vs $14.2 \%$ (p <0.001); to streptomycin $2.4 \%$ vs $12.5 \%$ (p <0.001); to rifampicin $0.9 \%$ vs $5 \%$ (p <0.05); to ethambutol $0.3 \%$ vs $2.5 \%(\mathrm{p}<0.05)$; and multiresistance $0.3 \%$ vs $2.5 \%$ (p $<0.05)$.

Conclusion. There are significant differences between local and immigrant populations in the pattern of resistances of the strains isolated. The immigrant population, due to the high frequency with which resistance is shown to isoniazid, must be treated initially with a pattern of four drugs until the result of the biogram is available.

Key words. M. tuberculosis. Resistance. Immigration.

\section{An. Sist. Sanit. Navar. 2009; 32 (2): 243-248}

1. Servicio de Microbiología. Hospital de Navarra. Pamplona.

2. Servicio de Microbiología. Hospital Virgen del Camino. Pamplona.

3. Instituto de Salud Pública. Pamplona. CIBER de Epidemiología y Salud Pública.

Recepción: 5 de marzo de 2009

Aceptación provisional: 7 de abril de 2009

Aceptación definitiva: 8 de junio de 2009

\section{Correspondencia}

Ana Navascués Ortega

Servicio de Microbiología

Hospital de Navarra

Irunlarrea, 3

31008 Pamplona

Tfno. 848422245

E-mail: ana.navascues.ortega@cfnavarra.es 


\section{INTRODUCCIÓN}

La tuberculosis es una enfermedad vinculada principalmente a la pobreza y común en los países de donde proceden la mayoría de inmigrantes que vienen a España. En los últimos años, debido al fenómeno de la migración, la epidemiología de la tuberculosis en Europa está cambiando. En 2006 los casos de tuberculosis en inmigrantes suponían una media del $20 \%$ de los casos diagnosticados; en algunos países de nuestro entorno llegaba a superar el $40 \%$ (Francia, Alemania, Italia) mientras que en otros como en España se aproximaba al $20 \%{ }^{1}$.

Dentro de nuestro país existen diferencias entre las distintas regiones en función del mayor o menor flujo de inmigrantes que presentan, habiéndose notado en la última década un aumento importante. En Navarra, la tasa de incidencia de tuberculosis ha disminuido en los últimos años y es una de las más bajas de España ${ }^{2}$; ha disminuido tanto en población autóctona como en inmigrante $(6,5 \%$ y $9,3 \%$ anual respectivamente), desde el año 2000 , con una tasa de incidencia por cada 100.000 habitantes del 9,9 y 51,5 en el año $2006^{3}$.

En los últimos años, debido al aumento de la inmigración en Navarra $(1,69 \%$ de la población en $2000,9,23 \%$ en $2007^{4}$ ) se ha producido un incremento en el número total de cepas de $M$. tuberculosis complex en esta población ${ }^{5}$; esto ha significado un cambio en la epidemiología de la tuberculosis y en su patrón de resistencias, ya que los inmigrantes proceden de países con no sólo mayores tasas de incidencia, sino también mayores porcentajes de resistencia ${ }^{6}$.

El control de la tuberculosis se basa en un diagnóstico temprano y en un adecuado tratamiento, por lo que es importante realizar una vigilancia periódica de las sensibilidades para adecuar el tratamiento ${ }^{7,8}$.

El objetivo de este trabajo es revisar la frecuencia de las resistencias en Navarra a los cuatro principales fármacos antituberculosos en población autóctona e inmigrante.

\section{MATERIAL Y MÉTODOS}

Se han estudiado los antibiogramas de 457 cepas de $M$. tuberculosis complex realizados en el Servicio de Microbiología del Hospital de Navarra durante el periodo 2000-2007. En este servicio se procesan las muestras de nuestra área y las de los hospitales comarcales de Estella y Tudela (aproximadamente 360.000 habitantes) y desde el año 2006 se reciben también las del Hospital Virgen del Camino, con lo que todos los antibiogramas de nuestra comunidad están centralizados (población estimada 600.000 habitantes).

El estudio de sensibilidad se llevó a cabo mediante el sistema radiométrico BACTEC460TB, estudiando la sensibilidad a los cuatro principales fármacos antituberculosos: estreptomicina $2 \mu \mathrm{g} / \mathrm{mL}$ (STRP), isoniacida $0,1 \mu \mathrm{g} / \mathrm{mL}$ (INH), rifampicina $2 \mu \mathrm{g} / \mathrm{mL}$ (RIF) y etambutol $2,5 \mu \mathrm{g} / \mathrm{mL}$ (ETM).

Para valorar la representatividad de los datos obtenidos, éstos se compararon con los casos de tuberculosis registrados en el sistema de vigilancia epidemiológica activa de Navarra durante el mismo periodo. Se consideró autóctonos a los nacidos en España e inmigrantes a los nacidos en otros países. Las comparaciones de porcentajes se realizaron mediante la prueba de $\chi^{2}$.

\section{RESULTADOS}

De las 457 cepas estudiadas, el 26,3\% fueron de inmigrantes, observándose un incremento significativo $(\mathrm{p}<0,05)$ a lo largo del periodo estudiado (Tabla 1).

El origen geográfico de la población inmigrante fue en el $36,6 \%$ de África (ÁfricaMagreb 28,3\% y África-Subsahariana 8,3\%), en el $35 \%$ de Sudamérica, en el $14,2 \%$ de Europa Occidental, en el 9,2\% de Europa del Este y en el 5\% de Asia. Los países de origen que destacan con un mayor número de casos fueron Marruecos, Ecuador y Portugal (Tabla 2). 
Tabla 1. Distribución del número de cepas en función del año y lugar de origen.

\begin{tabular}{|c|c|c|c|c|c|c|c|c|c|}
\hline & 2000 & 2001 & 2002 & 2003 & 2004 & 2005 & 2006 & 2007 & Total \\
\hline Autóctonos & $\begin{array}{c}57 \\
(82,6 \%)\end{array}$ & $52(80 \%)$ & $\begin{array}{c}37 \\
(78,7 \%)\end{array}$ & $\begin{array}{c}32 \\
(72,7 \%)\end{array}$ & $\begin{array}{c}40 \\
(70,2 \%)\end{array}$ & $\begin{array}{c}34 \\
(69,4 \%)\end{array}$ & $\begin{array}{c}44 \\
(67,7 \%)\end{array}$ & $\begin{array}{c}41 \\
(67,2 \%)\end{array}$ & $\begin{array}{c}337 \\
(73,7 \%)\end{array}$ \\
\hline Inmigrantes & $\begin{array}{c}12 \\
(17,4 \%)\end{array}$ & $13(20 \%)$ & $\begin{array}{c}10 \\
(21,3 \%)\end{array}$ & $\begin{array}{c}12 \\
(26,2 \%)\end{array}$ & $\begin{array}{c}17 \\
(29,8 \%)\end{array}$ & $\begin{array}{c}15 \\
(30,6 \%)\end{array}$ & $\begin{array}{c}21 \\
(32,3 \%)\end{array}$ & $\begin{array}{c}20 \\
(32,8 \%)\end{array}$ & $\begin{array}{c}120 \\
(26,3 \%)\end{array}$ \\
\hline Total & 69 & 65 & 47 & 44 & 57 & 49 & 65 & 61 & 457 \\
\hline
\end{tabular}

Tabla 2. País de origen de los inmigrantes.

\begin{tabular}{lclc}
\hline \multicolumn{1}{c}{ País } & $\mathbf{n}^{\mathbf{0}}$ de cepas & \multicolumn{1}{c}{ País } & $\mathbf{n}^{\mathbf{o}}$ de cepas \\
\hline Marruecos & 30 & G. Ecuatorial & 2 \\
Ecuador & 21 & Nigeria & 2 \\
Portugal & 14 & Bulgaria & 2 \\
Bolivia & 7 & Alemania & 2 \\
Rumania & 7 & Rusia & 1 \\
Perú & 5 & Bosnia & 1 \\
Brasil & 5 & Rep. Dominicana & 1 \\
Senegal & 5 & Lituania & 1 \\
Pakistán & 4 & China & 1 \\
Argelia & 4 & Camerún & 1 \\
Colombia & 2 & Filipinas & 1 \\
\hline & Total & & $\mathbf{1 1 9}^{*}$ \\
\hline
\end{tabular}

* Se desconoce el país de Sudamérica del que era originaria una de las pacientes

La resistencia al menos a un fármaco antituberculoso se detectó en el $5,6 \%$ y $20,8 \%(\mathrm{p}<0,001)$ de las cepas de la población autóctona e inmigrante respectivamente. Entre la población inmigrante, la mayor prevalencia se observó en los casos de Europa del Este $(36,4 \%)$ y la menor en los de África-Magreb (11,8\%).

Los porcentajes totales de resistencia a los distintos fármacos antituberculosos en población autóctona e inmigrante, respectivamente fueron: isoniacida $4,5 \%$ y $14,2 \%$ ( $<<0,001)$, rifampicina $0,9 \%$ y $5 \%(\mathrm{p}<0,05)$, etambutol $0,3 \%$ y $2,5 \%(\mathrm{p}<0,05)$ y estreptomicina $2,4 \%$ y $12,5 \%(\mathrm{p}<0,001)$. En este periodo se detectaron 4 cepas multirresistentes, un $0,3 \%$ de las cepas de población autóctona y un $2,5 \%$ de las de inmigrantes $(p<0,05)$. Una de estas cepas se aisló en un español "sin techo" con infección por VIH y las otras tres en inmigrantes. De estos últimos, 2 eran pacientes seronegativos a VIH que habían sido diagnosticados y tratados de tuberculosis previamente en su país (Colombia y Lituania) y el tercero era un paciente portugués con infección por VIH al que el año anterior se le había aislado una cepa de $M$. tuberculosis complex resistente a rifampicina. Por regiones, Europa del Este presentó los mayores índices de resistencia a isoniacida y estreptomicina $(27,3 \%)$ y etambutol $(9,1 \%)$. El menor porcentaje de resistencia a isoniacida (5,9\%) se encontró en Europa Occidental que a su vez tuvo el mayor a rifampicina $(17,6 \%)$. Hay que decir que todas las resistencias encontradas en esta región correspondieron a cepas de pacientes originarios de Portugal (Tabla 3). 
Tabla 3. Resistencias a los cuatro fármacos antituberculosos en población autóctona e inmigrante (2000-2007).

\begin{tabular}{|c|c|c|c|c|c|c|c|c|c|c|c|c|}
\hline & $\begin{array}{c}\text { Total } \\
\text { Antibiogramas }\end{array}$ & $\begin{array}{c}\text { Resistencia al } \\
\text { menos a un } \\
\text { antituberculoso }\end{array}$ & INH & RIF & STRP & INH+STRP & $\begin{array}{c}\mathrm{INH}+\mathrm{RIF} \\
+ \\
\mathrm{ETM}+\mathrm{STRP} \\
\end{array}$ & $\begin{array}{l}\text { Total } \\
\text { INH }\end{array}$ & $\begin{array}{l}\text { Total } \\
\text { RIF }\end{array}$ & Total ETM & Total STRP & $\begin{array}{l}\text { Total } \\
\text { MR }\end{array}$ \\
\hline Autóctonos & $\begin{array}{c}337 \\
(73,7 \%)\end{array}$ & $\begin{array}{c}19 \\
(5,6 \%)\end{array}$ & $\begin{array}{c}10 \\
(3,0 \%)\end{array}$ & $\begin{array}{c}2 \\
(0,6 \%)\end{array}$ & $\begin{array}{c}3 \\
(0,9 \%)\end{array}$ & $\begin{array}{c}4 \\
(1,2 \%)\end{array}$ & $\begin{array}{c}1 \\
(0,3 \%)\end{array}$ & $\begin{array}{c}15 \\
(4,5 \%)\end{array}$ & $\begin{array}{c}3 \\
(0,9 \%)\end{array}$ & $\begin{array}{c}1 \\
(0,3 \%)\end{array}$ & $\begin{array}{c}8 \\
(2,4 \%)\end{array}$ & $\begin{array}{c}1 \\
(0,3 \%)\end{array}$ \\
\hline Inmigrantes & $\begin{array}{c}120 \\
(26,3 \%)\end{array}$ & $\begin{array}{c}25 \\
(20,8 \%)\end{array}$ & $\begin{array}{c}7 \\
(5,8 \%)\end{array}$ & $\begin{array}{c}3 \\
(2,5 \%)\end{array}$ & $\begin{array}{c}5 \\
(4,2 \%)\end{array}$ & $\begin{array}{c}7 \\
(5,8 \%)\end{array}$ & $\begin{array}{c}3 \\
(2,5 \%)\end{array}$ & $\begin{array}{c}17 \\
(14,2 \%)\end{array}$ & $\begin{array}{c}6 \\
(5,0 \%)\end{array}$ & $\begin{array}{c}3 \\
(2,5 \%)\end{array}$ & $\begin{array}{c}15 \\
(12,5 \%)\end{array}$ & $\begin{array}{c}3 \\
(2,5 \%)\end{array}$ \\
\hline Sudamérica & $\begin{array}{c}42 \\
(35,0 \%)\end{array}$ & $\begin{array}{c}10 \\
(23,8 \%)\end{array}$ & $\begin{array}{c}3 \\
(7,1 \%)\end{array}$ & $\begin{array}{c}1 \\
(2,4 \%)\end{array}$ & $\begin{array}{c}3 \\
(7,1 \%)\end{array}$ & $\begin{array}{c}2 \\
(4,8 \%)\end{array}$ & $\begin{array}{c}1 \\
(2,4 \%)\end{array}$ & $\begin{array}{c}6 \\
(14,3 \%)\end{array}$ & $\begin{array}{c}2 \\
(4,8 \%)\end{array}$ & $\begin{array}{c}1 \\
(2,4 \%)\end{array}$ & $\begin{array}{c}6 \\
(14,3 \%)\end{array}$ & $\begin{array}{c}1 \\
(2,4 \%)\end{array}$ \\
\hline África-Magreb & $\begin{array}{c}34 \\
(28,3 \%)\end{array}$ & $\begin{array}{c}4 \\
(11,8 \%)\end{array}$ & $\begin{array}{c}2 \\
(5,9 \%)\end{array}$ & 0 & 0 & $\begin{array}{c}2 \\
(5,9 \%)\end{array}$ & 0 & $\begin{array}{c}4 \\
(11,8 \%)\end{array}$ & 0 & 0 & $\begin{array}{c}2 \\
(5,9 \%)\end{array}$ & 0 \\
\hline $\begin{array}{c}\text { Europa } \\
\text { Occidental }\end{array}$ & $\begin{array}{c}17 \\
(14,2 \%)\end{array}$ & $\begin{array}{c}4 \\
(23,5 \%)\end{array}$ & 0 & $\begin{array}{c}2 \\
(11,8 \%)\end{array}$ & $\begin{array}{c}1 \\
(5,9 \%)\end{array}$ & 0 & $\begin{array}{c}1 \\
(5,9 \%)\end{array}$ & $\begin{array}{c}1 \\
(5,9 \%)\end{array}$ & $\begin{array}{c}3 \\
(17,6 \%)\end{array}$ & $\begin{array}{c}1 \\
(5,9 \%)\end{array}$ & $\begin{array}{c}2 \\
(11,8 \%)\end{array}$ & $\begin{array}{c}1 \\
(5,9 \%)\end{array}$ \\
\hline Europa Este & $\begin{array}{c}11 \\
(9,2 \%)\end{array}$ & $\begin{array}{c}4 \\
(36,4 \%)\end{array}$ & $\begin{array}{c}1 \\
(9,1 \%)\end{array}$ & 0 & $\begin{array}{c}1 \\
(9,1 \%)\end{array}$ & $\begin{array}{c}1 \\
(9,1 \%)\end{array}$ & $\begin{array}{c}1 \\
(9,1 \%)\end{array}$ & $\begin{array}{c}3 \\
(27,3 \%)\end{array}$ & $\begin{array}{c}1 \\
(9,1 \%)\end{array}$ & $\begin{array}{c}1 \\
(9,1 \%)\end{array}$ & $\begin{array}{c}3 \\
(27,3 \%)\end{array}$ & $\begin{array}{c}1 \\
(9,1 \%)\end{array}$ \\
\hline $\begin{array}{c}\text { África- } \\
\text { Subsahariana }\end{array}$ & $\begin{array}{c}10 \\
(8,3 \%)\end{array}$ & $\begin{array}{c}2 \\
(20,0 \%)\end{array}$ & $\begin{array}{c}1 \\
(10,0 \%)\end{array}$ & 0 & 0 & $\begin{array}{c}1 \\
(10,0 \%)\end{array}$ & 0 & $\begin{array}{c}2 \\
(20,0 \%)\end{array}$ & 0 & 0 & $\begin{array}{c}1 \\
(10,0 \%)\end{array}$ & 0 \\
\hline Asia & $\begin{array}{c}6 \\
(5,0 \%)\end{array}$ & $\begin{array}{c}1 \\
(16,7 \%)\end{array}$ & 0 & 0 & 0 & $\begin{array}{c}1 \\
(16,7 \%)\end{array}$ & 0 & $\begin{array}{c}1 \\
(16,7 \%)\end{array}$ & 0 & 0 & $\begin{array}{c}1 \\
(16,7 \%)\end{array}$ & 0 \\
\hline
\end{tabular}

* MR: Multirresistencia = Resistencia a Isoniazida y Rifampicina.

Tabla 4. Resistencias totales a los cuatro fármacos por año y grupo de población.

\begin{tabular}{|c|c|c|c|c|c|c|c|c|c|c|}
\hline & Resistencia & $\begin{array}{l}2000 \\
n=57\end{array}$ & $\begin{array}{l}2001 \\
n=52\end{array}$ & $\begin{array}{l}2002 \\
n=37\end{array}$ & $\begin{array}{l}2003 \\
n=32\end{array}$ & $\begin{array}{l}2004 \\
n=40\end{array}$ & $\begin{array}{l}2005 \\
n=34\end{array}$ & $\begin{array}{l}2006 \\
n=44\end{array}$ & $\begin{array}{l}2007 \\
n=41\end{array}$ & Total \\
\hline \multirow{5}{*}{$\begin{array}{c}\text { Autóctonos } \\
\mathrm{n}=337\end{array}$} & Total INH & $\begin{array}{c}6 \\
10,5 \%\end{array}$ & $\begin{array}{c}4 \\
7,7 \%\end{array}$ & 0 & 0 & $\begin{array}{c}2 \\
5,0 \%\end{array}$ & 0 & $\begin{array}{c}1 \\
2,3 \%\end{array}$ & $\begin{array}{c}2 \\
4,9 \%\end{array}$ & $\begin{array}{c}15 \\
4,5 \%\end{array}$ \\
\hline & Total RIF & 0 & $\begin{array}{c}1 \\
1,6 \%\end{array}$ & 0 & $\begin{array}{c}1 \\
3,1 \%\end{array}$ & 0 & 0 & $\begin{array}{c}1 \\
2,3 \%\end{array}$ & 0 & $\begin{array}{c}3 \\
0,9 \%\end{array}$ \\
\hline & Total ETM & 0 & 0 & 0 & 0 & 0 & 0 & $\begin{array}{c}1 \\
2,27 \%\end{array}$ & 0 & $\begin{array}{c}1 \\
0,29 \%\end{array}$ \\
\hline & Total STRP & $\begin{array}{c}2 \\
3,5 \%\end{array}$ & 0 & $\begin{array}{c}1 \\
2,7 \%\end{array}$ & 0 & $\begin{array}{c}2 \\
5,0 \%\end{array}$ & 0 & $\begin{array}{c}1 \\
2,3 \%\end{array}$ & $\begin{array}{c}2 \\
4,9 \%\end{array}$ & $\begin{array}{c}8 \\
2,4 \%\end{array}$ \\
\hline & Resistencia & $\begin{array}{l}2000 \\
n=12\end{array}$ & $\begin{array}{l}2001 \\
n=13\end{array}$ & $\begin{array}{l}2002 \\
n=10\end{array}$ & $\begin{array}{l}2003 \\
n=12\end{array}$ & $\begin{array}{l}2004 \\
n=17\end{array}$ & $\begin{array}{l}2005 \\
n=15\end{array}$ & $\begin{array}{l}2006 \\
n=21\end{array}$ & $\begin{array}{l}2007 \\
n=20\end{array}$ & Total \\
\hline \multirow{4}{*}{$\underset{n=120}{\text { Inmigrantes }}$} & Total INH & $\begin{array}{c}1 \\
8,3 \%\end{array}$ & $\begin{array}{c}3 \\
23,1 \%\end{array}$ & $\begin{array}{c}2 \\
20,0 \%\end{array}$ & $\begin{array}{c}1 \\
8,3 \%\end{array}$ & $\begin{array}{c}2 \\
23,5 \%\end{array}$ & $\begin{array}{c}1 \\
6,7 \%\end{array}$ & $\begin{array}{c}4 \\
19,1 \%\end{array}$ & $\begin{array}{c}3 \\
15,0 \%\end{array}$ & $\begin{array}{c}17 \\
14,2 \%\end{array}$ \\
\hline & Total RIF & $\begin{array}{c}3 \\
25,0 \%\end{array}$ & $\begin{array}{c}1 \\
7,7 \%\end{array}$ & 0 & 0 & 0 & $\begin{array}{c}1 \\
6,7 \%\end{array}$ & 0 & $\begin{array}{c}1 \\
5,0 \%\end{array}$ & $\begin{array}{c}6 \\
5,0 \%\end{array}$ \\
\hline & Total ETM & $\begin{array}{c}1 \\
8,3 \%\end{array}$ & $\begin{array}{c}1 \\
7,7 \%\end{array}$ & 0 & 0 & 0 & $\begin{array}{c}1 \\
6,7 \%\end{array}$ & 0 & 0 & $\begin{array}{c}3 \\
2,5 \%\end{array}$ \\
\hline & Total STRP & $\begin{array}{c}2 \\
16,7 \%\end{array}$ & $\begin{array}{c}3 \\
23,1 \%\end{array}$ & $\begin{array}{c}3 \\
30,0 \%\end{array}$ & $\begin{array}{c}1 \\
8,3 \%\end{array}$ & $\begin{array}{c}2 \\
23,5 \%\end{array}$ & $\begin{array}{c}2 \\
13,3 \%\end{array}$ & $\begin{array}{c}1 \\
4,8 \%\end{array}$ & $\begin{array}{c}1 \\
5,0 \%\end{array}$ & $\begin{array}{c}15 \\
12,5 \%\end{array}$ \\
\hline
\end{tabular}


A lo largo del periodo, los datos de resistencia han variado tal como queda reflejado en la tabla 4. En la población autóctona, en el año 2000 y 2001 se produjeron los mayores picos de resistencia a isoniacida con un $10,5 \%$ y un $7,7 \%$. En los años posteriores, el porcentaje fue menor o no se registraron casos.

Las 457 cepas analizadas representaron el $65 \%$ de los 698 casos de tuberculosis registrados en Navarra durante el mismo periodo. Las cepas aisladas en población autóctona representaron el $67 \%$ y las aisladas en inmigrantes el $61 \%$.

\section{DISCUSIÓN}

En el periodo estudiado se ha producido un incremento en el número y porcentaje de aislamientos de $M$. tuberculosis de población inmigrante, pasando de un $17,4 \%$ en 2000 a un $32,8 \%$ en 2007. Éste es un hecho que se recoge en distintos trabajos españoles, y puede explicarse por el progresivo aumento de la inmigración en la última década ${ }^{9-11}$.

En un estudio nacional de resistencias en $M$. tuberculosis realizado en 2006 un $33,3 \%$ de las cepas pertenecían a pacientes inmigrantes ${ }^{12}$ dado que la inmigración no es un fenómeno que se haya producido de forma homogénea en todo el territorio español, es importante realizar estudios locales de vigilancia. Así, existen regiones como en El Ejido (Almería) ${ }^{13}$ donde ha llegado a suponer más del $70 \%$ en el año 2001 y otras como en la comarca del Bajo Deba (Guipúzcoa) ${ }^{14}$ donde en el periodo 19952006 supuso únicamente un $1,5 \%$ o en el Hospital de Sagunto (Valencia) un 2,6\% en el periodo 1999-2004 ${ }^{15}$.

En informes internacionales de la $\mathrm{OMS}^{5}$ se refleja, entre otros datos, los mayores porcentajes de resistencia de $M$. tuberculosis complex en los países de donde proceden la gran mayoría de los inmigrantes. En nuestro estudio se observa como las resistencias en esta población son significativamente superiores a las de la población autóctona.

La resistencia al menos a un fármaco antituberculoso en ambas poblaciones fue del 5,6\% en autóctonos frente al 20,8\% en inmigrantes. Esta diferencia queda reflejada también en otros trabajos españoles: $17,5 \%$ frente al $30,1 \%$ de Aznar y col ${ }^{16}, 3,7 \%$ frente al $13,1 \%$ de Tirado Balaguer y col$^{10}$ o el $7,5 \%$ frente al $13,6 \%$ de Ramos y col ${ }^{11}$ Destaca el 36,4\% de resistencia encontrado en la zona de Europa del Este lo que manifiesta el grave problema que presenta esta región.

Los casos analizados suponen una buena representación de la situación de la tuberculosis en Navarra, ya que han recogido el $65 \%$ de todos los casos registrados durante el periodo de estudio. Esto puede explicarse porque no siempre en todos los diagnósticos de tuberculosis se da el aislamiento de la micobacteria.

En cuanto a las resistencias totales los mayores porcentajes se encontraron a isoniacida y estreptomicina, tanto solas como combinadas, lo que puede deberse al amplio uso que se les ha dado ${ }^{17}$. El 4,5\% de resistencia a isoniacida en población autóctona es debido principalmente a 10 cepas resistentes encontradas en los dos primeros años de estudio pues suponen el $66,7 \%$ del total de cepas resistentes encontradas en este grupo. En cuanto al 14,2\% de resistencia a isoniacida en población inmigrante, la mayor o menor tasa de resistencia viene definida por el lugar de origen ya que el porcentaje máximo de resistencia se dio en Europa del Este y el menor en Europa Occidental. En este último todas las resistencias se dieron en pacientes portugueses que suponen la mayor parte del grupo.

En resumen, es necesario realizar estudios de vigilancia locales y, a la vista de los resultados obtenidos, es conveniente el tratamiento inicial con cuatro antituberculostáticos a los pacientes inmigrantes hasta disponer del resultado del antibiograma.

\section{BIBLIOGRAFÍA}

1. Surveillance of Tuberculosis in EuropeEuroTB. Report on tuberculosis cases notified in 2006. www.eurotb.org

2. Centro Nacional de Epidemiología. Comentario epidemiológico de las enfermedades de 
declaración obligatoria y sistema de información microbiológica. Año 2005. Bol Epidemiol Semanal 2006; 14: 193-200.

3. Castilla J, García Cenoz M, Irisarri F, Egüés N, Arriazu M, Barricarte A. Epidemiología de la tuberculosis en Navarra. An Sist Sanit Navar 2007; 30 Suppl 2: 21-32.

4. Instituto Navarro de Estadística. www.cfnavarra.es/estadistica

5. Castilla J, Urtiaga M, Hueto J, Sola J, DorronSORO I, ToRroba L et al. Evolución de las características epidemiológicas de la tuberculosis en Navarra (1994-2003). An Sist Sanit Navar 2005; 28: 237-245.

6. WHO Report № 4 . Anti-tuberculosis drug resistance in the world 2002-2007.

7. Boldú J, Cebollero P, Abu J, De Prado A. Tratamiento de la tuberculosis pulmonar. An Sist Sanit Navar 2007; 30 (Suppl 2): 99-115.

8. Dorronsoro I, Ojer M, Ruz A, Navascues A, GasTESI C, SALICIO Y. Estudio de la resistencia de Mycobacterium tuberculosis a los principales tuberculostáticos (Hospital de Navarra 19962003). An Sist Sanit Navar 2005; 28: 29-34.

9. Esteban J, Granizo JJ, Alvarez-Castillo MC, Soriano F. Drug resistance among Mycobacterium tuberculosis strains in immigrants: is there a real threat everywhere? Clin Microbiol Infect 2004; 10: 335-336.

10. Tirado Balaguer MD, Moreno Muñoz R, Marín Royo M, González Morán F, Pardo Serrano F, García del Busto Remón A et al. Impacto de la inmigración en las resistencias de Mycobacterium tuberculosis en la provincia de Caste- llón: 1995-2003. Med Clin (Barc) 2006; 126 : 761-764.

11. Ramos JM, Masiá M, Rodríguez JC, Padilla I, SoLER MJ, GuTIÉRREZ F. Tuberculosis en inmigrantes: diferencias clinicoepidemiológicas con la población autóctona (1999-2002). Enferm Infecc Microbiol Clin 2004; 22: 315-318.

12. Jiménez M, CASAl M. Grupo Español de Micobacteriología (GEM). Situación de las resistencias a fármacos de Mycobacterium tuberculosis en España. Rev Esp Quimioter 2008; 21: 22-25.

13. Díez F, Rogado M, Álvarez R, Delgado M, Hidalgo MA, SALAS J. Tuberculosis e inmigración en el poniente almeriense. Impacto en la incidencia y efectividad del tratamiento. Enferm Infecc Microbiol Clin 2002; 20 (Supl. 1): 191.

14. García-Zamalloa AM, Arrizabalaga J. Tuberculosis en la Comarca del Bajo Deba (Guipúzcoa) en el periodo 1995-2006. Enferm Infecc Microbiol Clin 2008; 26: 187-193.

15. Aragó Galindo M, Belda Mira A, Albert Contell A, Serra Sanchís B, Amorós Quiles I, Prat ForNELls J et al. Resistencias de Mycobacterium tuberculosis en el área del Hospital de Sagunto desde 1999 a 2004. Rev Clin Esp 2006; 206 : 376-381.

16. Aznar E, Domingo D, Abanades S, García-Peñuela E, LóPEz-Brea M. Resistencia en Mycobacterium tuberculosis durante un periodo de cuatro años en un hospital de Madrid. Rev Esp Quimioter 2005; 18: 222-225.

17. RichaRd LONG. Drug resistance tuberculosis. Can Med Assoc J 2000; 163: 425-428. 